\title{
Global solutions to vortex density equations arising from sup-conductivity
}

\section{Existence de solutions globales pour des équations de la super-conductivité}

\author{
Nader Masmoudi ${ }^{a, *}$, Ping Zhang ${ }^{\mathrm{b}}$ \\ ${ }^{a}$ Courant Institute, New York University, New York, NY 10012, USA \\ ${ }^{\mathrm{b}}$ Academy of Mathematics \& System Sciences, CAS, Beijing 100080, China
}

Received 6 July 2004; accepted 12 July 2004

Available online 7 April 2005

\begin{abstract}
In the first part of this paper, we establish the existence of a global renormalized solution to a family of vortex density equations arising from superconductivity. And we show by an explicit example the necessity of the notion of renormalized solution to be used here. In the second part, we prove the global existence and uniqueness of $W^{1, p}$ and $C^{\alpha}$ solutions to a modified model, which is derived from the physically sign-changing vortices case.
\end{abstract}

(C) 2005 L'Association Publications de l'Institut Henri Poincaré. Published by Elsevier B.V. All rights reserved

\section{Résumé}

On montre l'existence de solutions globales pour une famille d'équations provenant de la super-conductivité. On montre par un exemple que la notion de solutions renormalizées est nécessaire ici. Dans la seconde partie de ce papier, on montre l'existence et l'unicité de solutions $W^{1, p}$ et $C^{\alpha}$ pour un modèle qui décrit des vortex qui changent de signes.

(C) 2005 L'Association Publications de l'Institut Henri Poincaré. Published by Elsevier B.V. All rights reserved

MSC: 35Q35; 35M10

Keywords: Superconductivity; Vortex density; Hydrodynamics; Young measure; Renormalized solutions

\footnotetext{
* Corresponding author.

E-mail addresses: masmoudi@cims.nyu.edu (N. Masmoudi), zp@amss.ac.cn (P. Zhang).
} 


\section{Introduction}

This paper deals with two models coming from the hydrodynamic equations of Ginzburg-Landau vortices (see [13,6] for some earlier related works). In the first part of this paper, we shall establish the global existence of renormalized solutions to

$$
\left\{\begin{array}{l}
\partial_{t} \rho+\operatorname{div}(u \rho)=0, \quad(t, x) \in(0, \infty) \times \mathbb{R}^{2}, \\
u=\nabla \Delta^{-1} \rho, \\
\left.\rho\right|_{t=0}=\rho_{0},
\end{array}\right.
$$

with initial data $\rho_{0} \in L^{1}\left(\mathbb{R}^{2}\right)$.

Our main motivation to study this problem comes from the type-II superconductivity. It is generally accepted that, when effects due to thermal or field fluctuations are taken into account, the Abrikosov vortex lattice obtained from the mean-field theory can melt and form a vortex liquid. Then one of the important issues that one wishes to understand is the intrinsic nonlinear effects in the dynamics of such a liquid, where the vortex density satisfies (1.1). The rigorous finite gradient vortex dynamics was studied in [12] ( see also [10]). The formal derivation of (1.1) from the finite vortex dynamics was carried out in [19] (see also [1]). Under the assumption that $\rho_{0}$ is a positive Randon measure, the authors in [13] mathematically justified the formal derivation. One can check more physical explanation to (1.1) from $[19,1,13]$.

When we take a complex time relaxation in the finite gradient dynamics into account, we need to rotate the second equation of (1.1), and then the equation is modified to the following form:

$$
\left\{\begin{array}{l}
\partial_{t} \rho+\operatorname{div}(u \rho)=0, \quad(t, x) \in(0, \infty) \times \mathbb{R}^{2}, \\
u=\left(\begin{array}{cc}
\cos \theta & -\sin \theta \\
\sin \theta & \cos \theta
\end{array}\right) \nabla \Delta^{-1} \rho, \\
\left.\rho\right|_{t=0}=\rho_{0} .
\end{array}\right.
$$

Indeed, when $\cos \theta=0,(1.2)$ is the classical 2-D vorticity-formulated incompressible Euler equation. In that case, with smooth initial data, (1.2) has a unique global smooth solution. When $\rho_{0} \in L^{\infty}$, Yudovich [21] solves the global existence and uniqueness of weak solutions to (1.2). In [5] and [18], the authors establish the global existence of weak solutions to (1.2) with $\rho_{0} \in L^{p}$ for $1 \leqslant p<\infty$. However, the uniqueness of the weak solutions in this class is still open. When $\rho_{0} \in \mathcal{M}\left(\mathbb{R}^{2}\right) \cap H_{\text {loc }}^{-1}\left(\mathbb{R}^{2}\right)$, the above problem is the so-called vortex sheets problem in fluid mechanics. In 1991, Delort [7] solved the existence problem when $\rho_{0}$ keeps the sign, the remaining case is still an outstanding open question in the mathematical fluid mechanics.

Compared to the 2-D incompressible Euler equations, when $\cos \theta \neq 0$, smooth solution to (1.2) may blow up in finite time. In [13], the authors proved a global existence result to (1.1) when $\rho_{0} \in \mathcal{M}^{+}\left(\mathbb{R}^{2}\right)$. Furthermore, they found that $\rho(t, x)$ will be a function for $t>0$ and belongs to $L_{\text {loc }}^{p}\left(\mathbb{R}^{+} \times \mathbb{R}^{2}\right)$ with $p<2$. However, when $\rho_{0}$ changes sign, the second author and his collaborator [6] found that there exists concentration phenomena in the approximate solutions sequence of (1.2) no matter how smooth the initial data is. This argument implies the global existence of a measure-valued solution to (1.2). This motivates us to think that: to make the measure-valued solution more precise, we may need the notion of renormalized solution, which was first introduced by the DiPerna and Lions [3,4] in the study of transport and kinetic equations, for (1.2). On the other hand, it is easy to observe that (1.2) will keep the $L^{1}$ norm of $\rho$ nondecreasing with respect to $t$ even after formation of singularities to the smooth solution. Hence it is natural to study the global existence for the system (1.2) with initial data in $L^{1}$. As we allow $\rho_{0}$ to change sign, the proof of the global existence of the renormalized solution to (1.2) makes no difference for $\cos \theta \neq 0$. For simplicity, we take $\cos \theta=1$, which reduces to (1.1).

Note that the notion of renormalized solution can allow concentration in the solution, but it will make the problem much more nonlinear than the original problem. Then the main issue in the proof of the existence is to prove that there is no oscillation in the approximate solution sequence. Motivated by [14,15,22] and [11], we will use $L^{p}$ Young measure theory (see [20,17] and [16]) to cancel the possible oscillations in the approximate solutions. 
Then based on a space-time estimate for the approximate solutions in [6], in Section 2, we shall establish the existence of a global renormalized solution to (1.1) with initial data in $L^{1}$. Moreover, we will show by an example the necessity of the notion of renormalized solutions to be used here.

Considering the vortices of different sign and taking the London approximation to the induced magnetic field into account, a similar but modified system to (1.1) was derived in [1]:

$$
\left\{\begin{array}{l}
\partial_{t} \rho+\operatorname{div}(u|\rho|)=0, \quad(t, x) \in(0, \infty) \times \mathbb{R}^{2}, \\
u=\nabla\left(\lambda^{2} \Delta-I\right)^{-1} \rho, \\
\left.\rho\right|_{t=0}=\rho_{0} .
\end{array}\right.
$$

Here $\lambda$ denotes the penetration depth. A vector version of (1.3) was also available in [1] to take the three dimensional effect into account. When $\rho$ is a $\mathbb{R}^{2}$ valued function, using the stream function, the authors in [8] establish the existence and uniqueness of viscosity solution to an equation similar to (1.3). Such a technique obviously can not be used for the scalar case. Besides the stationary solutions studied in [1], we have not seen the other general existence result to (1.3).

To draw the main feature from (1.3) and get an analogy with (1.1), we consider the following system instead of (1.3):

$$
\left\{\begin{array}{l}
\partial_{t} \rho+\operatorname{div}(u|\rho|)=0, \quad(t, x) \in(0, \infty) \times \mathbb{R}^{2} \\
u=\nabla \Delta^{-1} \rho \\
\left.\rho\right|_{t=0}=\rho_{0}
\end{array}\right.
$$

In this case, when $\rho_{0}$ changes sign, we cannot prove the existence of $C^{1}$ solutions to (1.4) due to the fact that the term $|\rho|$ is only Lipschitz with respect to $\rho$. In Section 3, we decompose $\rho$ into a positive and a negative part, and use a time semi-discretization scheme to establish the global existence of solutions to (1.4) with initial data in $W^{1, p}$ and $C^{\alpha}$ for $2<p<\infty$ and $0<\alpha<1$ respectively. Hence we almost get the solution in the best possible space, which we can have for (1.4). It should be noted that the approach to be used here is completely different from the vanishing viscosity method in [6], where the authors proved a global existence result to the reduced one space dimensional case with initial data in $B V$.

Finally we point out that although (1.1) and (1.4) are derived in $\mathbb{R}^{2}$, our approach here does not depend too much on two space dimension. The arguments in this paper actually implies the corresponding result for the same equations in the general space dimension.

The outline of this paper is the following: in Section 2, we present the global existence of renormalized solutions to (1.1), and in Section 3, we prove the global existence and uniqueness of both $W^{1, p}$ and $C^{\alpha}$ solutions to (1.4).

\section{Global renormalized solutions to (1.1)}

In this section, we are going to establish the global existence of renormalized solutions to (1.1) with initial data in $L^{1}\left(\mathbb{R}^{2}\right)$. It is standard that the first step in the proof of the global existence of weak solutions is to construct the approximate solutions sequence. Note that given sign-changing smooth initial data $\rho_{0}$, it is easy to observe that the smooth solution to (1.1) will blow-up in finite time. Therefore to construct the approximate solutions to (1.1), we first introduce the following cut-off function

$$
\mathcal{T}_{\epsilon}(\xi):= \begin{cases}\xi, & \xi \geqslant-\frac{1}{\epsilon}, \\ -\frac{1}{\epsilon}, & \xi \leqslant-\frac{1}{\epsilon},\end{cases}
$$

and mollify the initial data $\rho_{0}$ by $\rho_{0, \epsilon}=\left(\rho_{0} \chi_{\epsilon}\right) * j_{\epsilon}$, where $\chi_{\epsilon}(x)=\chi(\epsilon x), \chi \in C_{c}^{\infty}\left(\mathbb{R}^{2}\right)$,

$$
\chi(x)= \begin{cases}1, & |x| \leqslant 1 \\ 0, & |x| \geqslant 2\end{cases}
$$


and $j_{\epsilon}$ is the standard Friedrich's mollifier with $\operatorname{supp} j_{\epsilon} \subset B_{\epsilon}(0)$, namely $j_{\epsilon}(x)=\frac{1}{\epsilon^{2}} j\left(\frac{x}{\epsilon}\right), j \in C_{c}^{\infty}\left(\mathbb{R}^{2}\right)$, and $\int j(x) \mathrm{d} x=1$. We consider

$$
\left\{\begin{array}{l}
\partial_{t} \rho_{\epsilon}+u_{\epsilon} \cdot \nabla \rho_{\epsilon}=-\mathcal{T}_{\epsilon}\left(\rho_{\epsilon}\right) \rho_{\epsilon}, \quad(t, x) \in(0, \infty) \times \mathbb{R}^{2}, \\
u_{\epsilon}=\nabla \Delta^{-1} \rho_{\epsilon} \\
\left.\rho_{\epsilon}\right|_{t=0}=\rho_{0, \epsilon}
\end{array}\right.
$$

Then by [6], (2.1) has a unique global smooth solution $\left(\rho_{\epsilon}, u_{\epsilon}\right)$ for any fixed $\epsilon$. Moreover, combining Lemma 2.1 and Lemma 2.2 of [6], we have

Lemma 2.1 (Solution of (2.1) with smooth data). Let $\rho_{0} \in L^{1}\left(\mathbb{R}^{2}\right)$. Then, for any fixed $\epsilon$, there exists a unique strong solution $\left(\rho_{\epsilon}, u_{\epsilon}\right)$ to $(2.1)$ such that $\rho_{\epsilon} \in L^{\infty}\left([0, T], W^{1, p}\left(\mathbb{R}^{2}\right)\right), \nabla u_{\epsilon} \in L^{\infty}\left([0, T], W^{1, p}\left(\mathbb{R}^{2}\right)\right)$ for any $1<p<\infty, T<\infty$, and

$$
\left\|\rho_{\epsilon}(t, \cdot)\right\|_{L^{1}} \leqslant\left\|\rho_{0}\right\|_{L^{1}}, \quad \rho(t, x) \leqslant \frac{1}{t} \quad \text { for } t>0 .
$$

Furthermore, for any $\alpha \in(0,1), T, L>0$, there exists a positive constant $C_{\alpha, T, L}$, which depends only on the $L^{1}$ norm of $\rho_{0}$ and the listed variables, such that

$$
\int_{0}^{T} \int_{|x| \leqslant L}\left|\rho_{\epsilon}\right|^{1+\alpha} \mathrm{d} x \mathrm{~d} t \leqslant C_{\alpha, T, L}
$$

Proof. For completeness, we outline the main idea of the proof here. One can check the proof of Lemma 2.2 of [6] for more details.

Step 1. Let $\alpha=d_{2} / d_{1} \in\left(0, \frac{1}{2}\right)$ with $d_{1}$ and $d_{2}$ being odd positive integers, and $\zeta(x) \in C_{c}^{\infty}\left(\mathbb{R}^{2}\right), \zeta \geqslant 0$ with $\zeta=1$ on $\{x|| x \mid \leqslant R\}$ and $\operatorname{supp} \zeta \subset\{x|| x \mid \leqslant R+1\}$. Set $\eta(\xi)=\alpha \int_{0}^{\xi} \max (1,|s|)^{\alpha-1} \mathrm{~d} s$ for $\xi \in \mathbb{R}^{1}$ such that $\eta^{\prime}(\xi)=\alpha \max (1,|s|)^{\alpha-1}$. We now multiply the first equation of $(2.1)$ by $\zeta(x) \eta^{\prime}\left(\rho_{\epsilon}\right)$, integrate the resulting identity over $[0, T] \times \mathbb{R}^{2}$, and perform integration by parts several times to obtain:

$$
\int_{0}^{T} \int_{\mathbb{R}^{2}} \zeta\left(\rho_{\epsilon} \eta\left(\rho_{\epsilon}\right)-\rho_{\epsilon} T_{\epsilon}\left(\rho_{\epsilon}\right) \eta^{\prime}\left(\rho_{\epsilon}\right)\right) \mathrm{d} x \mathrm{~d} t=\left.\int_{\mathbb{R}^{2}} \zeta \eta\left(\rho_{\epsilon}\right) \mathrm{d} x\right|_{0} ^{T}-\int_{0}^{T} \int_{\mathbb{R}^{2}} \nabla \zeta u_{\epsilon} \eta\left(\rho_{\epsilon}\right) \mathrm{d} x \mathrm{~d} s .
$$

Hence, by the definition of $\alpha$ and $\eta$, we have

$$
\int_{0}^{T} \int_{\mathbb{R}^{2}} \zeta\left(\rho_{\epsilon} \eta\left(\rho_{\epsilon}\right)-\rho_{\epsilon} T_{\epsilon}\left(\rho_{\epsilon}\right) \eta^{\prime}\left(\rho_{\epsilon}\right)\right) \mathrm{d} x \mathrm{~d} t \geqslant \int_{0}^{T} \int_{\mathbb{R}^{2}} 1_{\left|\rho_{\epsilon}\right| \geqslant 1 \zeta} \zeta\left((1-\alpha) \rho_{\epsilon}^{1+\alpha}+\alpha \rho_{\epsilon}\right) \mathrm{d} x,
$$

which together with the first part of (2.2), (2.4) and some classical estimates for $u_{\epsilon}$ leads to

$$
\int_{0}^{T} \int_{\left|\rho_{\epsilon}\right| \geqslant 1} \zeta \rho_{\epsilon}^{1+\alpha} \mathrm{d} x \mathrm{~d} t \leqslant \frac{1}{1-\alpha}\left(\alpha \int_{\mathbb{R}^{2}} \zeta\left|\rho_{\epsilon}\right| \mathrm{d} x+C_{1}+C_{2}\right),
$$

for all $\alpha=d_{2} / d_{1} \in\left(0, \frac{1}{2}\right)$.

Step 2. With (2.6), we take $\alpha=d_{2} / d_{1} \in\left(0, \frac{5}{6}\right)$, and repeat the argument from (2.4)-(2.6) to get

$$
\int_{0}^{T} \int_{\mid x \leqslant R+1}\left|\rho_{\epsilon}\right|^{p_{2}} \mathrm{~d} x \mathrm{~d} t \leqslant C\left(\alpha, R, T,\left\|\rho_{0, \epsilon}\right\|_{L^{1}}\right), \quad \forall p_{2}<\frac{11}{6} .
$$


Step 3. Inductively, we can prove that

$$
\int_{0}^{T} \int_{\mid x \leqslant R+1}\left|\rho_{\epsilon}\right|^{p_{n+1}} \mathrm{~d} x \mathrm{~d} t \leqslant C\left(\alpha, R, T,\left\|\rho_{0, \epsilon}\right\|_{L^{1}}\right), \quad \forall p_{n+1}<1+\alpha_{n+1},
$$

where $p_{n+1}=1+\alpha_{n}$, and $\alpha_{n}$ is defined by the inductive formula $\alpha_{n+1}=\left(1+3 \alpha_{n}\right) /\left(2\left(1+\alpha_{n}\right)\right)$. Note that $\lim _{n \rightarrow \infty} \alpha_{n}=1$, we complete the proof of (2.3).

Remark 2.1. We can also construct approximate solutions via the following system

$$
\left\{\begin{array}{l}
\partial_{t} \rho_{\epsilon}+\operatorname{div}\left(u_{\epsilon} \rho_{\epsilon}\right)=0, \quad(t, x) \in(0, \infty) \times \mathbb{R}^{2} \\
u_{\epsilon}=\nabla \Delta^{-1} \mathcal{T}_{\epsilon}\left(\rho_{\epsilon}\right) \\
\left.\rho_{\epsilon}\right|_{t=0}=\rho_{0, \epsilon}
\end{array}\right.
$$

Then we can also prove similar estimates for $\left(\rho_{\epsilon}, u_{\epsilon}\right)$ as that in (2.2) and (2.3).

From (2.2) and (2.3), there is a subsequence of $\left\{\rho_{\epsilon}\right\}$, which we denote $\left\{\rho_{\epsilon_{j}}\right\}$, and some function $\bar{\rho}(t, x) \in$ $L^{\infty}\left(\mathbb{R}^{+}, L^{1}\left(\mathbb{R}^{2}\right)\right) \cap L_{\text {loc }}^{p}\left(\mathbb{R}^{+} \times \mathbb{R}^{2}\right)$ for any $1<p<2$, such that

$$
\rho_{\epsilon_{j}} \rightarrow \bar{\rho} \quad \text { weakly in } L_{\text {loc }}^{p}\left(\mathbb{R}^{+} \times \mathbb{R}^{2}\right)
$$

as $\epsilon_{j} \rightarrow 0$. Moreover, by (2.2), (2.3) and a trivial interpolation, we find that

$\rho_{\epsilon}$ is uniformly bounded in $L_{\text {loc }}^{p_{1}}\left(\mathbb{R}^{+}, L_{\text {loc }}^{p_{2}}\left(\mathbb{R}^{2}\right)\right)$,

with $1 / p_{1}=\beta / q, 1 / p_{2}=1-\beta+\beta / q$ for all $0<\beta<1,1<q<2$. Therefore

$$
\left\{u_{\epsilon}\right\} \text { is uniformly bounded in } L_{\text {loc }}^{p_{1}}\left(\mathbb{R}^{+}, W_{\text {loc }}^{1, p_{2}}\left(\mathbb{R}^{2}\right)\right) \text {. }
$$

On the other hand, by (2.51) of [6], $\left\{\partial_{t} \rho_{\epsilon}\right\}$ is uniformly bounded in $L_{\text {loc }}^{p_{1}}\left(\mathbb{R}^{+}, W_{\text {loc }}^{-1,2 /(5-2 q)}\left(\mathbb{R}^{2}\right)+L^{1}\left(\mathbb{R}^{2}\right)\right)$. Then Lions-Aubin's Lemma implies that there is a subsequence of $\left\{u_{\epsilon}\right\}$, which we denote $\left\{u_{\epsilon_{j}}\right\}$, such that

$$
u_{\epsilon_{j}} \rightarrow u \triangleq \nabla \Delta^{-1} \bar{\rho} \quad \text { strongly in } L_{\mathrm{loc}}^{p_{1}}\left(\mathbb{R}^{+}, L_{\mathrm{loc}}^{s}\left(\mathbb{R}^{2}\right)\right),
$$

as $\epsilon_{j} \rightarrow 0$ and $s<p_{3}$ with $1 / p_{3}=1 / p_{2}-1 / 2$.

To prove that $(\bar{\rho}, u)$ thus obtained is indeed a weak solution to $(1.1)$, we need first to prove that there is no oscillation in the approximate solutions sequence. Arguing as in [14,15,22] and [11], we shall prove the precompactness of the solution sequence $\left\{\rho_{\epsilon}\right\}$ in $L^{p}([0, T] \times[-L, L])$ for any $0<T, L<\infty$ and $1<p<2$, by applying Young measure theory (see $[20,17]$ and [16]). For the convenience of the reader, we quote the following lemma from [11] (see also [5,9]).

Lemma 2.2 (Young measure). Let $\mathcal{U}$ be an open subset of $\mathbb{R}^{n}$, whose boundary has zero Lebesgue measure. Given a bounded family $\left\{v_{\epsilon}\right\} \subset L^{s}(\mathcal{U}), s>1$, of $\mathbb{R}^{N}$-valued functions, then there exist a subsequence $\left\{\epsilon_{j}\right\}$ and a measurable family of probability measure on $\mathbb{R}^{N},\left\{\mu_{y}(\cdot), y \in \mathcal{U}\right\}$, such that for all continuous functions $F(\lambda)$ with $F(\lambda)=O\left(|\lambda|^{q}\right)$ as $|\lambda| \rightarrow \infty$ and $q<s$, there holds

$$
\lim _{\epsilon_{j} \rightarrow 0} \int_{\mathcal{U}} \phi(y) F\left(v_{\epsilon_{j}}\right) \mathrm{d} y=\int_{\mathcal{U}} \phi(y) \int_{\mathbb{R}^{N}} F(\lambda) \mathrm{d} \mu_{y}(\lambda) \mathrm{d} y,
$$

for all $\phi(y) \in L^{r}(\mathcal{U})$ with compact support in the closure of $U$, where $1 / r+q / s=1$. Moreover,

$$
\iint|\lambda|^{s} \mathrm{~d} \mu_{y}(\lambda) \mathrm{d} y \leqslant \underline{\lim _{\epsilon_{j} \rightarrow 0}}\left\|v_{\epsilon_{j}}\right\|_{L^{s}}^{s}
$$


In the sequel, we will denote the weak limit of $F\left(v_{\epsilon}\right)$ by $\overline{F(v)}$ for convenience.

Combining Lemma 2.1 with Lemma 2.2, there is a family of Young measure $\mu_{t, x}(\lambda)$, such that for all continuous functions $F(\lambda)$ with $F(\lambda)=O\left(|\lambda|^{q}\right)$ as $|\lambda| \rightarrow \infty$ and $q<2$, there holds

$$
\lim _{\epsilon_{j} \rightarrow 0} \int_{\mathbb{R}^{+} \times \mathbb{R}^{2}} \phi(t, x) F\left(\rho_{\epsilon_{j}}\right) \mathrm{d} x \mathrm{~d} t=\int_{\mathbb{R}^{+} \times \mathbb{R}^{2}} \int_{\mathbb{R}} \phi(t, x) F(\lambda) \mathrm{d} \mu_{t, x}(\lambda) \mathrm{d} x \mathrm{~d} t,
$$

for all test function $\phi(t, x) \in C_{c}^{\infty}\left([0, \infty) \times \mathbb{R}^{2}\right)$. In particular, (2.9) and (2.15) imply that

$$
\bar{\rho}(t, x)=\int_{\mathbb{R}} \lambda \mathrm{d} \mu_{t, x}(\lambda) .
$$

With the above preparation, we will prove the precompactness of $\left\{\rho_{\epsilon}\right\}$.

Lemma 2.3 (precompactness of $\left.\left\{\rho_{\epsilon}\right\}\right)$. Let $\rho_{0} \in L^{1}\left(\mathbb{R}^{2}\right)$, then $\mu_{t, x}(\lambda)=\delta_{\bar{\rho}(t, x)}(\lambda)$.

Proof. The proof is based on an argument of the propagation of precompactness (see [14] and [15] for some similar arguments). As in [22], we separate the analysis of the precompactness of the solution sequence into the precompactness of the positive part and of the negative one respectively. Therefore, we decompose $\rho_{\epsilon}$ into

$$
\rho_{\epsilon}=\rho_{\epsilon} 1_{\rho_{\epsilon} \geqslant 0}+\rho_{\epsilon} 1_{\rho_{\epsilon} \leqslant 0} \triangleq \rho_{+, \epsilon}-\rho_{-, \epsilon},
$$

where $1_{\rho_{\epsilon} \geqslant 0}$ denote the characteristic function on the set $\left\{(t, x): \rho_{\epsilon}(t, x) \geqslant 0\right\}$, and so for $1_{\rho_{\epsilon} \leqslant 0}$.

Step 1. The propagation of the precompactness of the positive part of $\rho_{\epsilon}$.

Let us denote $\omega_{\epsilon}=\sqrt{\rho_{+, \epsilon}}$, by (2.3), $\left\{\omega_{\epsilon}\right\}$ is actually uniformly bounded in $L^{\infty}\left(\mathbb{R}^{+}, L^{2}\left(\mathbb{R}^{2}\right)\right) \cap L_{\text {loc }}^{q_{1}}\left(\mathbb{R}^{+} \times \mathbb{R}^{2}\right)$ for any $q_{1}<4$. Therefore by Lemma 2.2 , there is a subsequence of $\left\{\omega_{\epsilon}\right\}$ which we denote $\left\{\omega_{\epsilon_{j}}\right\}$, some function $\bar{\omega}(t, x) \in L^{\infty}\left(\mathbb{R}^{+}, L^{2}\left(\mathbb{R}^{2}\right)\right) \cap L_{\text {loc }}^{q_{1}}\left(\mathbb{R}^{+} \times \mathbb{R}^{2}\right)$, and a family of Young measure $v_{t, x}^{1}(\lambda)$, such that

$$
\omega_{\epsilon_{j}} \rightarrow \bar{\omega}=\int_{0}^{\infty} \lambda \mathrm{d} v_{t, x}^{1}(\lambda) \quad \text { weakly in } L^{\infty}\left(\mathbb{R}^{+}, L^{2}\left(\mathbb{R}^{2}\right)\right) \cap L_{\text {loc }}^{q_{1}}\left(\mathbb{R}^{+} \times \mathbb{R}^{2}\right),
$$

as $\epsilon_{j} \rightarrow 0$. Furthermore, a similar equality to that of (2.13) holds for the weak limit of $F\left(\omega_{\epsilon_{j}}\right)$ and $v_{t, x}^{1}(\lambda)$.

Next let us prove that $v_{t, x}^{1}(\lambda)=\delta_{\bar{\omega}(t, x)}(\lambda)$. Note that $\omega_{\epsilon}$ is only uniformly bounded in $L^{\infty}\left(\mathbb{R}^{+}, L^{2}\left(\mathbb{R}^{2}\right)\right)$, to study the propagation of the precompactness of $\omega_{\epsilon}$, we cannot take $F(\lambda)$ growing like $\left(O|\lambda|^{2}\right)$ at infinity. To overcome this technical difficulty, let us take the cut-off functions

$$
T_{R}(\xi)=\left\{\begin{array}{ll}
0, & \xi \leqslant 0, \\
\xi, & 0 \leqslant \xi \leqslant R, \\
R, & \xi \geqslant R,
\end{array} \quad S_{R}(\xi)= \begin{cases}0, & \xi \leqslant 0, \\
\frac{\xi^{2}}{2}, & 0 \leqslant \xi \leqslant R, \\
R\left(\xi-\frac{R}{2}\right), & \xi \geqslant R .\end{cases}\right.
$$

Noticing that $\rho_{\epsilon} \omega_{\epsilon}=\mathcal{T}_{\epsilon}\left(\rho_{\epsilon}\right) \omega_{\epsilon}=\omega_{\epsilon}^{3}$, from (2.1), it is easy to observe that

$$
\partial_{t} \omega_{\epsilon}+\operatorname{div}\left(u_{\epsilon} \omega_{\epsilon}\right)=\frac{1}{2} \omega_{\epsilon}^{3},
$$

holds in the sense of distributions. Formally multiplying (2.18) by $T_{R}\left(\omega_{\epsilon}\right)$, we infer that

$$
\partial_{t} S_{R}\left(\omega_{\epsilon}\right)+\operatorname{div}\left(u_{\epsilon} S_{R}\left(\omega_{\epsilon}\right)\right)=\omega_{\epsilon}^{2}\left(S_{R}\left(\omega_{\epsilon}\right)-\frac{1}{2} \omega_{\epsilon} T_{R}\left(\omega_{\epsilon}\right)\right) .
$$


The rigorous justification of (2.19) can be done by mollifying (2.18) first, then using Lemma II.1 of [3] to take the mollifying coefficient to be 0 . As it is rather standard, we omit the details here. (See (2.15)-(2.19) of [22] for a similar argument).

Combining (2.12) with Lemma 2.2, we take $\epsilon \rightarrow 0$ in (2.19) to get

$$
\partial_{t} \overline{S_{R}(\omega)}+\operatorname{div}\left(u \overline{S_{R}(\omega)}\right)=\overline{\omega^{2}\left(S_{R}(\omega)-\frac{1}{2} \omega T_{R}(\omega)\right)} .
$$

On the other hand, again by (2.12), we take $\epsilon \rightarrow 0$ in (2.18) to find

$$
\partial_{t} \bar{\omega}+\operatorname{div}(u \bar{\omega})=\frac{1}{2} \overline{\omega^{3}} .
$$

In the sequel, we denote $\overline{\rho_{+}}, \overline{\rho_{-}}$the weak limits of $\rho_{+, \epsilon}$ and $\rho_{-, \epsilon}$ respectively. Then trivially $\overline{\rho_{+}}=\overline{\omega^{2}}$. Formally multiplying (2.21) by $T_{R}(\bar{\omega})$, a trivial calculation yields

$$
\partial_{t} S_{R}(\bar{\omega})+\operatorname{div}\left(u S_{R}(\bar{\omega})\right)=\left(\overline{\omega^{2}}-\overline{\rho_{-}}\right)\left(S_{R}(\bar{\omega})-T_{R}(\bar{\omega}) \bar{\omega}\right)+\frac{1}{2} \overline{\omega^{3}} T_{R}(\bar{\omega})
$$

where we have used the fact that $\operatorname{div}(u)=\left(\overline{\omega^{2}}-\overline{\rho_{-}}\right)$.

Subtracting (2.22) from (2.20), we arrive at

$$
\begin{aligned}
\partial_{t}\left(\overline{S_{R}(\omega)}-S_{R}(\bar{\omega})\right)+\operatorname{div}\left(u\left(\overline{S_{R}(\omega)}-S_{R}(\bar{\omega})\right)\right) \\
\quad=\overline{\omega^{2}\left(S_{R}(\omega)-\frac{1}{2} \omega T_{R}(\omega)\right)}-\left(\overline{\omega^{2}}-\overline{\rho_{-}}\right)\left(S_{R}(\bar{\omega})-\frac{1}{2} T_{R}(\bar{\omega}) \bar{\omega}\right) \\
\quad-\frac{1}{2} \overline{\rho_{-}} T_{R}(\bar{\omega}) \bar{\omega}-\frac{1}{2} T_{R}(\bar{\omega})\left(\overline{\omega^{3}}-\overline{\omega^{2}} \bar{\omega}\right) .
\end{aligned}
$$

Note by the second inequality of (2.2), we have: $\omega_{\epsilon}, \bar{\omega} \leqslant 1 / \sqrt{t}$ for $t>0$. Therefore, noting that $S_{R}(\xi)=\frac{1}{2} \xi T_{R}(\xi)$ for $\xi \leqslant R$, we deduce that

$$
S_{R}^{+}\left(\omega_{\epsilon}\right)-\frac{1}{2} \omega_{\epsilon} T_{R}^{+}\left(\omega_{\epsilon}\right)=0=S_{R}(\bar{\omega})-\frac{1}{2} T_{R}(\bar{\omega}) \bar{\omega}
$$

for $t \geqslant \frac{1}{R^{2}}$, which together with Lemma 2.2 implies that

$$
\overline{\omega^{2}\left(S_{R}(\omega)-\frac{1}{2} \omega T_{R}(\omega)\right)}=0,
$$

for $t \geqslant 1 / R^{2}$. While the classical convexity inequality yields

$$
\overline{\omega^{3}} \geqslant \overline{\omega^{2}} \bar{\omega}
$$

which together with (2.23)-(2.25) implies that

$$
\partial_{t}\left(\overline{S_{R}(\omega)}-S_{R}(\bar{\omega})\right)+\operatorname{div}\left(u\left(\overline{S_{R}(\omega)}-S_{R}(\bar{\omega})\right)\right) \leqslant 0
$$

for $t \geqslant 1 / R^{2}$.

Let us denote $g=: \frac{1}{2}\left(\overline{\omega^{2}}-\bar{\omega}^{2}\right)$. Then a similar proof of (2.24) yields

$$
\left(\overline{S_{R}(\omega)}-S_{R}(\bar{\omega})\right)(t, x)=g(t, x)
$$

for a. e. $(t, x) \in\left(1 / R^{2}, \infty\right) \times \mathbb{R}^{2}$. Furthermore, there holds 


$$
\begin{aligned}
& \|g(t, \cdot)\|_{L^{1}} \leqslant \frac{1}{2} \int_{\mathbb{R}^{2}} \overline{\omega^{2}(t, x)} \mathrm{d} x \leqslant \frac{1}{2} \int_{\mathbb{R}^{2}}|\rho(t, x)| \mathrm{d} x, \\
& \|g(t, \cdot)\|_{L^{\infty}} \leqslant \frac{1}{2} \overline{\omega^{2}} \leqslant \frac{1}{2 t} .
\end{aligned}
$$

Now we are going to use the argument, which is used in the last step of proof to Lemma 3.2 of [24] and (6.39) of [22], to complete the proof of the propagation of the precompactness of the positive part of the approximate solutions sequence. First, from (2.18), it is easy to prove that

$$
\partial_{t} \omega_{\epsilon}^{2}+\operatorname{div}\left(u_{\epsilon} \omega_{\epsilon}^{2}\right)=0
$$

holds in the sense of distributions, therefore,

$$
\int_{\mathbb{R}^{2}} \omega_{\epsilon}^{2}(t, x) \mathrm{d} x \leqslant \int_{\mathbb{R}^{2}} \omega_{0, \epsilon}^{2} \mathrm{~d} x,
$$

which implies that

$$
\int_{\mathbb{R}^{2}} \bar{\omega}^{2}(t, x) \mathrm{d} x \leqslant \int_{\mathbb{R}^{2}} \overline{\omega^{2}}(t, x) \mathrm{d} x \leqslant \int_{\mathbb{R}^{2}} \bar{\omega}_{0}^{2} \mathrm{~d} x,
$$

as $\omega_{0, \epsilon}$ strongly converges to $\bar{\omega}_{0}$ in $L^{2}(\mathbb{R})$. While from (2.21), we obtain

$$
\bar{\omega}(t, x) \rightarrow \bar{\omega}_{0}(x) \quad \text { weakly in } L^{2}(\mathbb{R}) \text { as } t \rightarrow 0 .
$$

Hence by summing up (2.30) and (2.31), and using Theorem 1 of [9], we get

$$
\lim _{t \rightarrow 0} \int_{\mathbb{R}^{2}} \bar{\omega}^{2}(t, x) \mathrm{d} x=\int_{\mathbb{R}^{2}} \bar{\omega}_{0}^{2} \mathrm{~d} x .
$$

Combining (2.32) with (2.30), we arrive at

$$
\lim _{t \rightarrow 0} \int_{\mathbb{R}^{2}} \overline{\omega^{2}}(t, x) \mathrm{d} x \leqslant \int_{\mathbb{R}^{2}} \bar{\omega}_{0}^{2} \mathrm{~d} x \leqslant \lim _{t \rightarrow 0} \int_{\mathbb{R}^{2}} \bar{\omega}^{2}(t, x) \mathrm{d} x,
$$

which implies that

$$
\lim _{t \rightarrow 0} \int_{\mathbb{R}^{2}}\left(\overline{\omega^{2}}-\bar{\omega}^{2}\right)(t, x) \mathrm{d} x=0 .
$$

Furthermore, motivated by [24], let us take $\phi(x) \in C_{c}^{\infty}\left(\mathbb{R}^{2}\right)$ with $\phi(x)=1$ for $|x| \leqslant 1$ and $\phi(x)=0$ for $|x|>2$, and take $\delta=6 / R^{2}, \bar{t}>20 / R^{2}$ to be one of the Lebesgue points of $\int_{\mathbb{R}^{2}} g(t, x) \phi(x / n) \mathrm{d} x$, and take $\psi^{\delta}(t) \in C_{c}^{\infty}\left(1 / R^{2}, \infty\right)$ such that

$$
\begin{aligned}
& \psi^{\delta}(t)= \begin{cases}0, & t \leqslant \frac{\delta}{2} \text { or } t \geqslant \bar{t}+\delta, \\
1, & \delta \leqslant t \leqslant \bar{t}-\delta,\end{cases} \\
& 0 \leqslant \partial_{t} \psi^{\epsilon}(t) \leqslant \frac{C}{\delta}, \quad t \in[0, \delta], \quad-\partial_{t} \psi^{\epsilon}(t) \leqslant \frac{C}{\delta}, \quad t \in[\bar{t}-\delta, \bar{t}+\delta] .
\end{aligned}
$$

Let us multiply $\psi^{\delta}(t) \phi\left(\frac{x}{n}\right)$ to (2.26) and integrate the resulting inequality over $\left(\frac{\delta}{4}, \infty\right) \times \mathbb{R}^{2}$ to yield 


$$
\begin{aligned}
& \frac{C}{\delta} \int_{\bar{t}-\delta}^{\bar{t}+\delta} \int_{\mathbb{R}^{2}} g \phi\left(\frac{x}{n}\right) \mathrm{d} x \mathrm{~d} t \leqslant-\int_{\bar{t}-\delta}^{\bar{t}+\delta} \int_{\mathbb{R}^{2}} \partial_{t} \psi^{\delta} g \phi\left(\frac{x}{n}\right) \mathrm{d} x \mathrm{~d} t \\
& \quad \leqslant \int_{\delta / 4}^{\delta} \int_{\mathbb{R}^{2}} \partial_{t} \psi^{\delta} g \phi\left(\frac{x}{n}\right) \mathrm{d} x \mathrm{~d} t+\frac{1}{n} \int_{\delta / 4}^{\bar{t}+\delta} \int_{\mathbb{R}^{2}} \psi^{\delta} \nabla \phi\left(\frac{x}{n}\right) u g \mathrm{~d} x \mathrm{~d} t \\
& \quad \leqslant \frac{C}{\delta} \int_{\delta / 4}^{\delta} \int_{\mathbb{R}^{2}} g \phi\left(\frac{x}{n}\right) \mathrm{d} x \mathrm{~d} t-\frac{C}{n} \int_{\delta / 4}^{\bar{t}+\delta} \int_{\mathbb{R}^{2}} \psi^{\delta} \bar{\rho} \nabla \Delta^{-1}\left(\nabla \phi\left(\frac{x}{n}\right) g\right) \mathrm{d} x \mathrm{~d} t,
\end{aligned}
$$

where in the last step, we used integration by parts and the fact that $u=\nabla \Delta^{-1} \bar{\rho}$. To proceed further, note by the standard inequality in 2 space dimension that

$$
\left|\nabla \Delta^{-1} h\right|_{L^{\infty}} \leqslant C\|h\|_{L^{\infty}}^{1 / 2}\|h\|_{L^{1}}^{1 / 2}
$$

and (2.28), we find

$$
\mid \nabla \Delta^{-1}\left(\nabla \phi\left(\frac{x}{n}\right) g\right)\left\|_{L^{\infty}} \leqslant C\right\| \nabla \phi\left\|_{L^{\infty}}\right\| g\left\|_{L^{1}}^{1 / 2}\right\| g\left\|_{L^{\infty}}^{1 / 2} \leqslant C\right\| \nabla \phi \|_{L^{\infty}} t^{-1 / 2} .
$$

Plugging (2.35) to (2.34), and taking $R \rightarrow \infty$ in the resulting inequality, we find by (2.33) that

$$
C \int_{\mathbb{R}^{2}} g(\bar{t}, x) \phi\left(\frac{x}{n}\right) \mathrm{d} x \leqslant \frac{C}{n} \int_{0}^{\bar{t}} \int_{\mathbb{R}^{2}}|\bar{\rho}(t, x)|\|\nabla \phi\|_{L^{\infty}} t^{-1 / 2} \mathrm{~d} x \mathrm{~d} t \leqslant \frac{C \sqrt{\bar{t}}\|\nabla \phi\|_{L^{\infty}}}{n},
$$

which together Fatou's Lemma yields that

$$
\int_{\mathbb{R}^{2}} g(\bar{t}, x) \mathrm{d} x=0 .
$$

Note that $\int_{\mathbb{R}^{2}} g(t, x) \phi\left(\frac{x}{n}\right) \mathrm{d} x \in L^{\infty}\left(\mathbb{R}^{+}\right)$, therefore, almost all $t \in \mathbb{R}^{+}$is a Lebesgue point of $\int_{\mathbb{R}^{2}} g(t, x) \phi\left(\frac{x}{n}\right) \mathrm{d} x$. Due to the arbitrariness of $\bar{t}$, we obtain

$$
g(t, x)=0, \quad \text { a.e. }(t, x) \in \mathbb{R}^{+} \times \mathbb{R}^{2} .
$$

Hence for a.e. $(t, x) \in \mathbb{R}^{+} \times \mathbb{R}^{2}$, there holds

$$
\int_{\mathbb{R}^{2}} \int_{0}^{\infty}|\lambda-\bar{\omega}|^{2} \mathrm{~d} v_{t, x}^{1}(\lambda) \mathrm{d} x=\int_{\mathbb{R}^{2}}\left(\overline{\omega^{2}}-\bar{\omega}^{2}\right)(t, x) \mathrm{d} x=0,
$$

which implies that

$$
v_{t, x}^{1}(\lambda)=\delta_{\bar{\omega}(t, x)}(\lambda)
$$

for a. e. $(t, x) \in \mathbb{R}^{+} \times \mathbb{R}^{2}$.

Step 2. The proof of the precompactness for the negative part of $\rho_{\epsilon}$.

To prove the precompactness of the negative part of the solutions sequence $\left\{\rho_{-, \epsilon}\right\}$, we will use a different renormalization procedure to the approximate solutions sequence. Firstly by $(2.1), \rho_{-, \epsilon}$ satisfies

$$
\partial_{t} \rho_{-, \epsilon}+u_{\epsilon} \cdot \nabla \rho_{-, \epsilon}=-\mathcal{T}_{\epsilon}\left(\rho_{\epsilon}\right) \rho_{-, \epsilon},
$$


in the sense of distributions. We denote $\left(\rho_{-, \epsilon}\right)^{1 / 4}$ by $\eta_{\epsilon}$, then, by Lemma $2.1,\left\{\eta_{\epsilon}\right\}$ is uniformly bounded in $L^{\infty}\left(\mathbb{R}^{+}, L^{4}\left(\mathbb{R}^{2}\right)\right) \cap L_{\text {loc }}^{r}\left(\mathbb{R}^{+} \times \mathbb{R}^{2}\right)$ for any $r<8$. Therefore, by Lemma 2.2, there exists a subsequence of $\left\{\eta_{\epsilon}\right\}$, $\left\{\eta_{\epsilon_{j}}\right\}$, and a family of Young measure $v_{t, x}^{2}(\lambda)$ such that a similar equality as that of (2.13) holds for $\left\{\eta_{\epsilon_{j}}\right\}$ and $v_{t, x}^{2}(\lambda)$. Moreover, from (2.40), a trivial calculation yields

$$
\partial_{t} \eta_{\epsilon}+\operatorname{div}\left(u_{\epsilon} \eta_{\epsilon}\right)=-\eta_{\epsilon}^{5}+\frac{1}{4} T_{1 / \epsilon}\left(\eta_{\epsilon}^{4}\right) \eta_{\epsilon}
$$

Let us denote $\bar{\eta}(t, x)=\int_{0}^{\infty} \lambda \mathrm{d} v_{t, x}^{2}(\lambda)$. Then by taking $\epsilon \rightarrow 0$ in (2.41) and using (2.12), we find

$$
\partial_{t} \bar{\eta}+\operatorname{div}(u \bar{\eta})=-\frac{3}{4} \overline{\eta^{5}}
$$

While from (2.41), it is easy to observe that

$$
\partial_{t} \eta_{\epsilon}^{2}+\operatorname{div}\left(u_{\epsilon} \eta_{\epsilon}^{2}\right)=-\eta_{\epsilon}^{6}+\frac{1}{2} T_{1 / \epsilon}\left(\eta_{\epsilon}^{4}\right) \eta_{\epsilon}^{2}
$$

then similar to the proof of (2.42), we get

$$
\partial_{t} \overline{\eta^{2}}+\operatorname{div}\left(u \overline{\eta^{2}}\right)=-\frac{1}{2} \overline{\eta^{6}} .
$$

On the other hand, note that $\overline{\rho_{-}}=\overline{\eta^{4}},(2.42)$ together with an argument following (2.19) implies

$$
\partial_{t} \bar{\eta}^{2}+\operatorname{div}\left(u \bar{\eta}^{2}\right)=\left(\overline{\eta^{4}}-\overline{\rho_{+}}\right) \bar{\eta}^{2}-\frac{3}{2} \overline{\eta^{5}} \bar{\eta} .
$$

Subtracting (2.44) from (2.43), we arrive at

$$
\partial_{t}\left(\overline{\eta^{2}}-\bar{\eta}^{2}\right)+\operatorname{div}\left[u\left(\overline{\eta^{2}}-\bar{\eta}^{2}\right)\right]=-\frac{1}{2} \overline{\eta^{6}}-\overline{\eta^{4}} \bar{\eta}^{2}+\frac{3}{2} \overline{\eta^{5}} \bar{\eta}+\overline{\rho_{+}} \bar{\eta}^{2} .
$$

Notice that from (2.3) and (2.39), we can take a subsequence of $\left\{\rho_{+, \epsilon}\right\},\left\{\rho_{+, \epsilon_{j}}\right\}$, such that $\rho_{+, \epsilon_{j}} \rightarrow \overline{\rho_{+}}$in $L_{\text {loc }}^{p}\left(\mathbb{R}^{+} \times \mathbb{R}^{2}\right)$ for any $p<2$. Therefore,

$$
\rho_{+, \epsilon_{j}} \eta_{\epsilon_{j}} \rightarrow \overline{\rho_{+}} \bar{\eta} \quad \text { weakly in } L_{\text {loc }}^{s}\left(\mathbb{R}^{+} \times \mathbb{R}^{2}\right)
$$

for any $s<\frac{8}{5}$. But by their definitions, $\rho_{+, \epsilon_{j}} \eta_{\epsilon_{j}}=0$, which together with (2.46) implies that

$$
\overline{\rho_{+}} \bar{\eta}=0 \text {. }
$$

Hence, the right-hand side of (2.45) equals

$$
\begin{aligned}
-\frac{1}{2} \overline{\eta^{6}}-\overline{\eta^{4}} \bar{\eta}^{2}+\frac{3}{2} \overline{\eta^{5}} \bar{\eta} & =-\left(\frac{1}{2}\left(\overline{\eta^{6}}+\overline{\eta^{4}} \bar{\eta}^{2}\right)-\overline{\eta^{5}} \bar{\eta}\right)+\frac{1}{2}\left(\overline{\eta^{5}} \bar{\eta}-\overline{\eta^{4}} \bar{\eta}^{2}\right) \\
& =-\frac{1}{2} \int_{\mathbb{R}} \lambda^{4}(\lambda-\bar{\eta})^{2} \mathrm{~d} v_{t, x}^{2}(\lambda)+\frac{1}{2} \int_{\mathbb{R}} \lambda^{4} \bar{\eta}(\lambda-\bar{\eta}) \mathrm{d} v_{t, x}^{2}(\lambda),
\end{aligned}
$$

note that $\int_{\mathbb{R}} \bar{\eta}^{5}(\lambda-\bar{\eta}) \mathrm{d} v_{t, x}^{2}(\lambda)=0$, from which, we obtain

$$
\begin{aligned}
(2.48) & =-\frac{1}{2} \int_{\mathbb{R}} \lambda^{4}(\lambda-\bar{\eta})^{2} \mathrm{~d} v_{t, x}^{2}(\lambda)+\frac{1}{2} \int_{\mathbb{R}}\left(\lambda^{4}-\bar{\eta}^{4}\right) \bar{\eta}(\lambda-\bar{\eta}) \mathrm{d} v_{t, x}^{2}(\lambda) \\
& =\frac{1}{2} \int_{\mathbb{R}}\left(-\lambda^{4}+\lambda^{3} \bar{\eta}+\lambda^{2} \bar{\eta}^{2}+\lambda \bar{\eta}^{3}+\bar{\eta}^{4}\right)(\lambda-\bar{\eta})^{2} \mathrm{~d} v_{t, x}^{2}(\lambda) \\
& \leqslant C \bar{\eta}^{4} \int_{\mathbb{R}}(\lambda-\bar{\eta})^{2} \mathrm{~d} v_{t, x}^{2}(\lambda) .
\end{aligned}
$$


Combining (2.45) with (2.49), we obtain

$$
\partial_{t}\left(\overline{\eta^{2}}-\bar{\eta}^{2}\right)+\operatorname{div}\left(u\left(\overline{\eta^{2}}-\bar{\eta}^{2}\right)\right) \leqslant C \bar{\eta}^{4} \int_{\mathbb{R}}(\lambda-\bar{\eta})^{2} \mathrm{~d} v_{t, x}^{2}(\lambda) .
$$

Trivially

$$
\bar{\eta}^{4} \leqslant \overline{\eta^{4}}=\overline{\rho_{-}}, \quad \int_{\mathbb{R}}(\lambda-\bar{\eta})^{2} \mathrm{~d} v_{t, x}^{2}(\lambda)=\left(\overline{\eta^{2}}-\bar{\eta}^{2}\right),
$$

while a similar proof of (2.47) also implies

$$
\overline{\rho_{+}} \overline{\eta^{2}}=0,
$$

which together with (2.47) and (2.50) implies that

$$
\partial_{t}\left(\overline{\eta^{2}}-\bar{\eta}^{2}\right)+\operatorname{div}\left(u\left(\overline{\eta^{2}}-\bar{\eta}^{2}\right)\right) \leqslant C\left(\overline{\rho_{-}}-\overline{\rho_{+}}\right)\left(\overline{\eta^{2}}-\bar{\eta}^{2}\right)=-C \bar{\rho}\left(\overline{\eta^{2}}-\bar{\eta}^{2}\right),
$$

where we used the fact that $\bar{\rho}=-\left(\overline{\rho_{-}}-\overline{\rho_{+}}\right)$. In what follows, we denote $\left(\overline{\eta^{2}}-\bar{\eta}^{2}\right)$ by $f, f_{\epsilon}=f * j_{\epsilon}$, then by Lemma II.1 of [3], we obtain

$$
\partial_{t} f_{\epsilon}+\operatorname{div}\left(u f_{\epsilon}\right) \leqslant-C \bar{\rho} f_{\epsilon}+r_{\epsilon},
$$

with $r_{\epsilon} \rightarrow 0$ in $L_{\text {loc }}^{s}\left(\mathbb{R}^{+} \times \mathbb{R}^{2}\right)$ for $s<\frac{4}{3}$. Let us take $\theta, \gamma>0$ be small constants, which will be determined later. Then multiplying (2.52) by $\theta\left(f_{\epsilon}+\gamma\right)^{\theta-1}$, we find

$$
\partial_{t}\left(f_{\epsilon}+\gamma\right)^{\theta}+\operatorname{div}\left(u\left(f_{\epsilon}+\gamma\right)^{\theta}\right)=(\theta(C-1)-1)\left(\overline{\rho_{-}}-\overline{\rho_{+}}\right)\left(f_{\epsilon}+\gamma\right)^{\theta}+\theta\left(f_{\epsilon}+\gamma\right)^{\theta-1} r_{\epsilon} .
$$

Taking $\epsilon \rightarrow 0$ then $\gamma \rightarrow 0$ in (2.53), and picking the constant $\theta$ small enough such that $\theta(C-1)-1 \leqslant 0$ and $f^{\theta}(t, x) \in L^{\infty}\left(\mathbb{R}^{+}, L^{4}\left(\mathbb{R}^{2}\right)\right)$, we arrive at

$$
\partial_{t} f^{\theta}+\operatorname{div}\left(u f^{\theta}\right) \leqslant 0 .
$$

With (2.54), a similar proof of (2.34) and (2.36) implies that: for almost all $\bar{t} \in \mathbb{R}^{+}$, there holds

$$
C \int_{\mathbb{R}^{2}} f^{\theta}(\bar{t}, x) \phi\left(\frac{x}{n}\right) \mathrm{d} x \leqslant-\frac{C}{n} \int_{0}^{\bar{t}} \int_{\mathbb{R}^{2}} \bar{\rho}(t, x) \nabla \Delta^{-1}\left(\nabla \phi\left(\frac{x}{n}\right) f^{\theta}\right) \mathrm{d} x \mathrm{~d} t .
$$

On the other hand, note that

$$
\begin{aligned}
\left|\nabla \Delta^{-1} h\right| & =\left|\int_{\mathbb{R}^{2}} \frac{x-y}{|x-y|^{2}} h(y) \mathrm{d} y\right| \\
& \leqslant\left|\int_{|x-y| \leqslant r} \frac{x-y}{|x-y|^{2}} h(y) \mathrm{d} y\right|+\left|\int_{|x-y| \geqslant r} \frac{x-y}{|x-y|^{2}} h(y) \mathrm{d} y\right| \\
& \leqslant C\left(r^{1 / 2}\|h\|_{L^{4}}+\frac{1}{r}\|h\|_{L^{1}}\right) .
\end{aligned}
$$

By taking $r=\left(\|h\|_{L^{1}} /\|h\|_{L^{\infty}}\right)^{2 / 3}$ in the above inequality, we obtain

$$
\left\|\nabla \Delta^{-1} h\right\|_{L^{\infty}} \leqslant C\|h\|_{L^{1}}^{1 / 3}\|h\|_{L^{4}}^{2 / 3} .
$$

In particular, from (2.56), we obtain 


$$
\begin{aligned}
\left\|\nabla \Delta^{-1}\left(\nabla \phi\left(\frac{x}{n}\right) f^{\theta}\right)\right\|_{L^{\infty}} & \leqslant C\left\|\nabla \phi\left(\frac{x}{n}\right)\right\|_{L^{4 / 3}}^{1 / 3}\|\nabla \phi\|_{L^{\infty}}\left\|f^{\theta}\right\|_{L^{4}} \\
& \leqslant C n^{1 / 2}\|\nabla \phi\|_{L^{4 / 3}}^{1 / 3}\|\nabla \phi\|_{L^{\infty}}^{2 / 3}\left\|f^{\theta}\right\|_{L^{4}} .
\end{aligned}
$$

Plugging (2.57) to (2.55), and using a similar procedure as that in the proof of (2.37), we obtain

$$
\int_{\mathbb{R}^{2}} f^{\theta}(t, x) \mathrm{d} x=0,
$$

for almost all $t>0$. This implies that

$$
v_{t, x}^{2}(\lambda)=\delta_{\bar{\eta}(t, x)(\lambda)} .
$$

Combining (2.39) with (2.58), we complete the proof of the lemma.

Before the presentation of the main result of this section, let us first introduce the precise definition of the renormalized solution to (1.1).

Definition 2.1. We call $(\rho(t, x), u(t, x))$ a renormalized solution of $(1.1)$ if for any $\beta(\tau) \in C^{1}(\mathbb{R})$ with $\beta(0)=0$ and $\beta^{\prime}(\tau)=O\left(|\tau|^{\alpha-1}\right)$ for some $0<\alpha<1$, there holds

$$
\partial_{t} \beta(\rho)+\operatorname{div}(u \beta(\rho))=\rho \beta(\rho)-\rho^{2} \beta^{\prime}(\rho),
$$

and

$$
u=\nabla \Delta^{-1} \rho,
$$

in the sense of distributions.

Theorem 2.4. Let $\rho_{0} \in L^{1}\left(\mathbb{R}^{2}\right)$, then (1.1) has a global renormalized solution $(\rho, u)$ in the sense of Definition 2.1. Furthermore, $\rho(t, x) \in L^{\infty}\left(\mathbb{R}^{+}, L^{1}\left(\mathbb{R}^{2}\right)\right) \cap L_{\mathrm{loc}}^{q}\left(\mathbb{R}^{+} \times \mathbb{R}^{2}\right)$ for any $q<2$, and $u(t, x) \in L_{\mathrm{loc}}^{p_{1}}\left(\mathbb{R}^{+}, W_{\mathrm{loc}}^{1, p_{2}}\left(\mathbb{R}^{2}\right)\right)$ with the exponents $p_{1}, p_{2}$ given at the next line of (2.10). Furthermore, for $t>0$, there holds

$$
\rho(t, x)<\frac{1}{t}, \quad \text { a.e. } x \in \mathbb{R}^{2} .
$$

Remark 2.2. Note by (2.61) that there are only concentrations on the negative part of $\rho(t, x)$. Therefore, we actually only need to renormalize the negative part of $\rho$ in Definition 2.1.

Proof. We first construct the approximate solutions via (2.1). Then from (2.3), (2.11) and setting $\rho=\bar{\rho}$, we obtain $(\rho, u)$ with the required regularity as that stated in the theorem. Moreover, from (2.12), there holds (2.60). Therefore to complete the proof of the theorem, we only need to justify that $(\rho, u)$ thus obtained satisfies (2.59). In fact, by multiplying $\beta^{\prime}\left(\rho_{\epsilon}\right)$ to the first equation of (2.1), we get

$$
\partial_{t} \beta\left(\rho_{\epsilon}\right)+\operatorname{div}\left(u_{\epsilon} \beta\left(\rho_{\epsilon}\right)\right)=\rho_{\epsilon} \beta\left(\rho_{\epsilon}\right)-\mathcal{T}_{\epsilon}\left(\rho_{\epsilon}\right) \rho_{\epsilon} \beta^{\prime}\left(\rho_{\epsilon}\right)
$$

Note by (2.3) and Lemma 2.3, we find that there is a subsequence of $\left\{\rho_{\epsilon}\right\},\left\{\rho_{\epsilon_{j}}\right\}$, such that

$$
\rho_{\epsilon_{j}} \rightarrow \rho \quad \text { strongly } L_{\text {loc }}^{q}\left(\mathbb{R}^{+} \times \mathbb{R}^{2}\right)
$$

for any $q<2$. Then by taking $\epsilon=\epsilon_{j}$ in (2.62), and using (2.12), we prove (2.59). This completes the proof of the theorem. 
Remark 2.3. In the one space dimension case, (2.1) is reduced to

$$
\partial_{t} \rho+\partial_{x}(u \rho)=0, \quad u(t, x)=\int_{0}^{x} \rho(t, y) \mathrm{d} y .
$$

It is easy to observe that when $\rho(0, x)$ takes negative values, a smooth solution to (2.64) will blow up in finite time. In fact, we have the following explicit solution $(\rho, u)$ to $(2.64)$ :

$$
\rho(t, x)=\left\{\begin{array}{ll}
0, & t \geqslant 1, \\
-\frac{1}{1-t} \chi_{[t-1,1-t]}(x), & 0<t \leqslant 1, \\
-\chi_{[-1,1]}(x), & t=0,
\end{array} \quad u(t, x)= \begin{cases}0, & t \geqslant 1, \\
1, & x \leqslant t-1, \\
-\frac{x}{1-t}, & t-1 \leqslant x \leqslant 1-t, \\
-1, & x \geqslant 1-t .\end{cases}\right.
$$

On can easily check that $(\rho, u)$ thus defined is a renormalized solution but not a distributional weak solution to $(2.64)$.

\section{Global strong solutions to (1.4)}

In this section, we consider the global existence and uniqueness of strong solutions to (1.4) with initial data $\rho_{0}(x) \in L^{1}\left(\mathbb{R}^{2}\right) \cap W^{1, p}\left(\mathbb{R}^{2}\right)$ for $2<p<\infty$. Formally we decompose the solution $\rho$ of (1.4) into

$$
\rho=\rho 1_{\rho \geqslant 0}+\rho 1_{\rho \leqslant 0} \triangleq \rho_{+}-\rho_{-},
$$

where $1_{\rho} \geqslant 0$ again denotes the characteristic function on the set $\{(t, x): \rho(t, x) \geqslant 0\}$, and similar meaning for $1_{\rho \leqslant 0}$. Then we can rewrite the first equation of (1.4) as

$$
\partial_{t}\left(\rho_{+}-\rho_{-}\right)+u \cdot \nabla\left(\rho_{+}+\rho_{-}\right)=-\left(\rho_{+}\right)^{2}+\left(\rho_{-}\right)^{2} .
$$

Motivated by this formulation, for any fixed small constant $\epsilon>0$, we decompose the time interval $[0, \infty)$ as $\bigcup_{i=0}^{\infty}[i \epsilon,(i+1) \epsilon)$, and will construct the approximate solutions to (1.4) on each time interval $[i \epsilon,(i+1) \epsilon)$, then pitch them together to get the global approximate solutions. First, on the time interval $[0, \epsilon)$, we solve for $\left(\rho_{ \pm, 1}^{\epsilon}, u_{1}^{\epsilon}\right)$ through

$$
\left\{\begin{array}{l}
\partial_{t} \rho_{ \pm, 1}^{\epsilon} \pm u_{1}^{\epsilon} \cdot \nabla \rho_{ \pm, 1}^{\epsilon}=-\left(\rho_{ \pm, 1}^{\epsilon}\right)^{2}, \quad t \in[0, \epsilon], \\
u_{1}^{\epsilon}=\nabla \Delta^{-1}\left(\rho_{+, 1}^{\epsilon}-\rho_{-, 1}^{\epsilon}\right), \\
\left.\rho_{+, 1}^{\epsilon}\right|_{t=0}=\rho_{+, 0},\left.\quad \rho_{-, 1}^{\epsilon}\right|_{t=0}=\rho_{-, 0},
\end{array}\right.
$$

where $\rho_{+, 0}=\rho_{0} 1_{\rho_{0} \geqslant 0}$ and $\rho_{-, 0}=-\rho_{0} 1_{\rho_{0} \leqslant 0}$. As both $\rho_{+, 0}$ and $\rho_{-, 0}$ are positive functions, mollifying the initial data by $j_{\eta}$, we can use the classical characteristic method to solve the above problem globally. For any fixed $\eta$, we can get an $\eta$ independent estimate for the approximate solutions, then we take $\eta$ to 0 to get the estimate for the solutions of (3.2) with rough initial data. For simplicity, we will omit this step in the subsequence, and will do the a priori estimate directly.

By taking $\partial_{x_{i}}$ to the first equation of (3.2), and multiplying the resulting equation by $p\left|\partial_{x_{i}} \rho_{+, 1}^{\epsilon}\right|^{p-1} \operatorname{sign}\left(\partial_{x_{i}} \rho_{+, 1}^{\epsilon}\right)$, we obtain

$$
\begin{aligned}
& \partial_{t}\left|\partial_{x_{i}} \rho_{+, 1}^{\epsilon}\right|^{p}+\operatorname{div}\left(u_{1}^{\epsilon}\left|\partial_{x_{i}} \rho_{+, 1}^{\epsilon}\right|^{p}\right) \\
& \quad=\operatorname{div} u_{1}^{\epsilon}\left|\partial_{x_{i}} \rho_{+, 1}^{\epsilon}\right|^{p}-p \partial_{x_{i}} u_{1}^{\epsilon} \cdot \nabla \rho_{+, 1}^{\epsilon}\left|\partial_{x_{i}} \rho_{+, 1}^{\epsilon}\right|^{p-1} \operatorname{sign}\left(\partial_{x_{i}} \rho_{+, 1}^{\epsilon}\right)-2 p \rho_{+, 1}^{\epsilon}\left|\partial_{x_{i}} \rho_{+, 1}^{\epsilon}\right|^{p} .
\end{aligned}
$$

Note from the characteristic form of (3.2), it is trivial to observe that

$$
0 \leqslant \rho_{ \pm, 1}^{\epsilon}(t, x) \leqslant\left\|\rho_{0}\right\|_{L^{\infty}}, \quad\left\|\rho_{ \pm, 1}^{\epsilon}(t, \cdot)\right\|_{L^{1}} \leqslant\left\|\rho_{ \pm, 0}\right\|_{L^{1}} .
$$


Then integrating (3.3) over $\mathbb{R}^{2}$, and using Gronwall inequality and (3.4), we find

$$
\left\|\nabla_{x} \rho_{+, 1}^{\epsilon}(t, \cdot)\right\|_{L^{p}} \leqslant\left\|\nabla_{x} \rho_{+, 0}\right\|_{L^{p}} \mathrm{e}^{C \int_{0}^{t}\left\|\nabla_{x} u_{1}^{\epsilon}(s, \cdot)\right\|_{L^{\infty}} \mathrm{d} s} .
$$

Similar to the estimate of (3.5), we can prove the following estimate for $\nabla_{x} \rho_{-, 1}^{\epsilon}$

$$
\left\|\nabla_{x} \rho_{-, 1}^{\epsilon}(t, \cdot)\right\|_{L^{p}} \leqslant\left\|\nabla_{x} \rho_{-, 0}\right\|_{L^{p}} \mathrm{e}^{C \int_{0}^{t}\left\|\nabla_{x} u_{1}^{\epsilon}(s, \cdot)\right\|_{L^{\infty}} \mathrm{d} s} .
$$

This completes the construction and the estimate for the approximate solutions on the time interval $[0, \epsilon)$. To go to the next step, let us define the data at time $\epsilon$ first. At time $t=\epsilon$, we redesign $\rho_{+, 1}^{\epsilon}$ and $\rho_{-, 1}^{\epsilon}$ by setting

$$
\rho_{+, 1}^{\epsilon}(\epsilon+, x)=\left(\rho_{+, 1}^{\epsilon}-\rho_{-, 1}^{\epsilon}\right)_{+}(\epsilon-, x), \quad \rho_{-, 1}^{\epsilon}(\epsilon+, x)=\left(\rho_{-, 1}^{\epsilon}-\rho_{+, 1}^{\epsilon}\right)_{+}(\epsilon-, x) .
$$

With the above definition, it is easy to observe that

$$
\left\|\nabla_{x} \rho_{+, 1}^{\epsilon}(\epsilon+, x)\right\|_{L^{p}}+\left\|\nabla_{x} \rho_{-, 1}^{\epsilon}(\epsilon+, x)\right\|_{L^{p}} \leqslant\left\|\nabla_{x} \rho_{+, 1}^{\epsilon}(\epsilon-, x)\right\|_{L^{p}}+\left\|\nabla_{x} \rho_{-, 1}^{\epsilon}(\epsilon-, x)\right\|_{L^{p}} .
$$

Indeed, for any fixed positive constant $h$, we denote $D_{h}^{+}=\left|\rho_{+, 1}^{\epsilon}(\epsilon+, x+h)-\rho_{+, 1}^{\epsilon}(\epsilon+, x)\right|+\mid \rho_{-, 1}^{\epsilon}(\epsilon+, x+$ $h)-\rho_{-, 1}^{\epsilon}(\epsilon+, x) \mid$, and similar notation for $D_{h}^{-}$with $\epsilon+$ in $D_{h}^{+}$replaced by $\epsilon-$. Then, if $\rho_{+, 1}^{\epsilon}(\epsilon-, x+h) \geqslant$ $\rho_{-, 1}^{\epsilon}(\epsilon-, x+h)$, we have the following two subcases: either $\rho_{+, 1}^{\epsilon}(\epsilon-, x) \geqslant \rho_{-, 1}^{\epsilon}(\epsilon-, x)$, then

$$
D_{h}^{+}=\left|\rho_{+, 1}^{\epsilon}(\epsilon-, x+h)-\rho_{-, 1}^{\epsilon}(\epsilon-, x+h)-\rho_{+, 1}^{\epsilon}(\epsilon-, x)+\rho_{-, 1}^{\epsilon}(\epsilon-, x)\right| \leqslant D_{h}^{-},
$$

or $\rho_{+, 1}^{\epsilon}(\epsilon-, x) \leqslant \rho_{-, 1}^{\epsilon}(\epsilon-, x)$, and then

$$
D_{h}^{+}=\left(\rho_{+, 1}^{\epsilon}(\epsilon-, x+h)-\rho_{-, 1}^{\epsilon}(\epsilon-, x+h)\right)+\left(\rho_{-, 1}^{\epsilon}(\epsilon-, x)-\rho_{+, 1}^{\epsilon}(\epsilon-, x)\right) \leqslant D_{h}^{-} .
$$

While when $\rho_{+, 1}^{\epsilon}(\epsilon-, x+h) \leqslant \rho_{-, 1}^{\epsilon}(\epsilon-, x+h)$, similar to the proof of (3.9) and (3.10), we still can prove that $D_{h}^{+} \leqslant D_{h}^{-}$.

Combining (3.9) with (3.10), we obtain

$$
\int_{\mathbb{R}^{2}}\left(\frac{D_{h}^{+}}{h}\right)^{p} \mathrm{~d} x \leqslant \int_{\mathbb{R}^{2}}\left(\frac{D_{h}^{-}}{h}\right)^{p} \mathrm{~d} x,
$$

taking $h \rightarrow 0$ in the above inequality, we get (3.8).

Next we solve for $\left(\rho_{ \pm, 2}^{\epsilon}, u_{2}^{\epsilon}\right)$ on $[\epsilon, 2 \epsilon)$ by (3.2) with $\left(\rho_{ \pm, 1}^{\epsilon}, u_{1}^{\epsilon}\right)$ there replaced by $\left(\rho_{ \pm, 2}^{\epsilon}, u_{2}^{\epsilon}\right)$, together with the data $\rho_{ \pm, 1}^{\epsilon}(\epsilon+, x)$ at $t=\epsilon$. From (3.4) and its proof, it is easy to get that

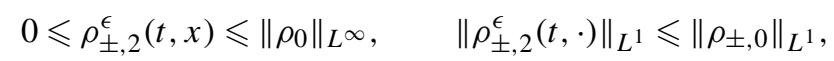

for $\epsilon \leqslant t<2 \epsilon$.

Furthermore, similar to the proof of (3.5) and (3.6), we can get similar estimates for $\left\|\nabla_{x} \rho_{ \pm, 2}^{\epsilon}\right\|_{L^{p}}$.

With the above argument, we can inductively define the approximate solutions on $[i \epsilon,(i+1) \epsilon)$ for any integer $i$. And on each time step, there hold similar estimates as (3.5), (3.6) and (3.8). Now we define the global approximate solutions to (1.4) by

$$
\left\{\begin{array}{l}
\rho_{ \pm}^{\epsilon}(t, x)=\rho_{ \pm, i+1}^{\epsilon}(t, x), \quad \text { for } t \in[i \epsilon,(i+1) \epsilon), \\
u^{\epsilon}(t, x)=u_{i}^{\epsilon}(t, x), \quad \text { for } t \in[i \epsilon,(i+1) \epsilon), \\
\rho^{\epsilon}(t, x)=\rho_{+}^{\epsilon}(t, x)-\rho_{-}^{\epsilon}(t, x) .
\end{array}\right.
$$

Then from (3.5), (3.6) and (3.8), we find

$$
\left\|\nabla_{x} \rho_{+}^{\epsilon}(t, \cdot)\right\|_{L^{p}}+\left\|\nabla_{x} \rho_{-}^{\epsilon}(t, \cdot)\right\|_{L^{p}} \leqslant\left(\left\|\nabla_{x} \rho_{+, 0}^{\epsilon}\right\|_{L^{p}}+\left\|\nabla_{x} \rho_{-, 0}^{\epsilon}\right\|_{L^{p}}\right) \mathrm{e}^{C \int_{0}^{t}\left\|\nabla_{x} u^{\epsilon}(s, \cdot)\right\|_{L^{\infty}} \mathrm{d} s},
$$

for all $t \in \mathbb{R}^{+}$. Therefore, from (3.12), we obtain

$$
\left\|\nabla_{x} \rho^{\epsilon}(t, \cdot)\right\|_{L^{p}} \leqslant 2\left\|\nabla_{x} \rho_{0}\right\|_{L^{p}} \mathrm{e}^{C \int_{0}^{t}\left\|\nabla_{x} u^{\epsilon}(s, \cdot)\right\|_{L^{\infty}} \mathrm{d} s} .
$$


On the other hand, note that $u^{\epsilon}=\nabla \Delta^{-1} \rho^{\epsilon}$, by Lemma 2.2 of [2], we have

$$
\left\|\nabla_{x} u^{\epsilon}(t, \cdot)\right\|_{L^{\infty}} \leqslant C\left\|\rho^{\epsilon}\right\|_{L^{\infty}} \log \left(1+\frac{\left\|\nabla_{x} \rho^{\epsilon}(t, \cdot)\right\|_{L^{p}}}{\left\|\rho^{\epsilon}\right\|_{L^{\infty}}}\right) .
$$

Summing up (3.4), (3.14) and (3.15), we arrive at

$$
\left\|\nabla_{x} \rho^{\epsilon}(t, \cdot)\right\|_{L^{p}} \leqslant\left\|\nabla_{x} \rho_{0}\right\|_{L^{p}} \mathrm{e}^{C \int_{0}^{t} \log \left(1+\left\|\nabla_{x} \rho^{\epsilon}(s, \cdot)\right\|_{L^{p}}\right) \mathrm{d} s} .
$$

To get the uniform estimate for $\left\|\nabla_{x} \rho^{\epsilon}(t, \cdot)\right\|_{L^{p}}$, let us set

$$
F_{\epsilon}(t)=\left\|\nabla_{x} \rho_{0}\right\|_{L^{p}} \mathrm{e}^{C \int_{0}^{t} \log \left(1+\left\|\nabla_{x} \rho^{\epsilon}(s, \cdot)\right\|_{L} p\right) \mathrm{d} s},
$$

then from (3.16), we get

$$
F_{\epsilon}^{\prime}(t)=C \log \left(1+\left\|\nabla_{x} \rho^{\epsilon}(t, \cdot)\right\|_{L^{p}}\right) F_{\epsilon}(t) \leqslant C \log \left(1+F_{\epsilon}(t)\right) F_{\epsilon}(t),
$$

from which, we obtain

$$
\left\|\nabla_{x} \rho^{\epsilon}(t, \cdot)\right\|_{L^{p}} \leqslant F_{\epsilon}(t) \leqslant\left\|\nabla_{x} \rho_{0}\right\|_{L^{p}} \mathrm{e}^{C \mathrm{e}^{C t}},
$$

which together with (3.13) and (3.15) implies that there is a positive constant $C(t)$, which is independent of $\epsilon$, such that

$$
\left\|\nabla_{x} \rho_{ \pm}^{\epsilon}(t, \cdot)\right\|_{L^{p}} \leqslant C(t) .
$$

Furthermore, by (3.4) and (3.11), we have

$$
\left\|\rho_{ \pm}^{\epsilon}(t, \cdot)\right\|_{L^{1} \cap L^{\infty}} \leqslant\left\|\rho_{ \pm, 0}\right\|_{L^{1} \cap L^{\infty}}
$$

which together with (3.19) and some basic fact on singular integral operator implies that

$$
\left\|u^{\epsilon}(t, \cdot)\right\|_{W^{2, p}} \leqslant C(t) \text {. }
$$

To prove the precompactness of $\left(\rho^{\epsilon}, u^{\epsilon}\right)$, we need also some weak continuity of $\rho^{\epsilon}$ with respect to the $t$ variable. In order to do so, for any positive constant $T<\infty$, let us take any test function $\phi(t, x) \in \mathcal{D}\left([0, T) \times \mathbb{R}^{2}\right)$. Denote $K=\left[\frac{T}{\epsilon}\right]$, the integer part of $\frac{T}{\epsilon}$. Then from the first equation of $(3.2)$, we find

$$
\begin{aligned}
& \int_{0}^{T} \int_{\mathbb{R}^{2}}\left(\partial_{t} \phi+u^{\epsilon} \cdot \nabla \phi-\rho_{-}^{\epsilon} \phi\right) \rho_{+}^{\epsilon} \mathrm{d} x \mathrm{~d} t \\
& \quad=\int_{K \epsilon \mathbb{R}^{2}}^{T}\left(\partial_{t} \phi+u^{\epsilon} \cdot \nabla \phi-\rho_{-}^{\epsilon} \phi\right) \rho_{+}^{\epsilon} \mathrm{d} x \mathrm{~d} t+\sum_{i=0}^{K-1} \int_{i \epsilon}^{(i+1) \epsilon} \int_{\mathbb{R}^{2}}\left(\partial_{t} \phi+u^{\epsilon} \cdot \nabla \phi-\rho_{-}^{\epsilon} \phi\right) \rho_{+}^{\epsilon} \mathrm{d} x \mathrm{~d} t \\
& \quad=\sum_{i=1}^{K} \int_{\mathbb{R}^{2}} \phi(i \epsilon, x)\left(\rho_{+, i}^{\epsilon}(i \epsilon-, x)-\rho_{+, i}^{\epsilon}(i \epsilon+, x)\right) \mathrm{d} x-\int_{\mathbb{R}^{2}} \phi(0, x) \rho_{+, 0}(x) \mathrm{d} x .
\end{aligned}
$$

Similar to the proof of (3.21), again from (3.2), we get

$$
\begin{aligned}
& \int_{0}^{T} \int_{\mathbb{R}^{2}}\left(\partial_{t} \phi-u^{\epsilon} \cdot \nabla \phi-\rho_{+}^{\epsilon} \phi\right) \rho_{-}^{\epsilon} \mathrm{d} x \mathrm{~d} t \\
& \quad=\sum_{i=1}^{K} \int_{\mathbb{R}^{2}} \phi(i \epsilon, x)\left(\rho_{-, i}^{\epsilon}(i \epsilon-, x)-\rho_{-, i}^{\epsilon}(i \epsilon+, x)\right) \mathrm{d} x-\int_{\mathbb{R}^{2}} \phi(0, x) \rho_{-, 0}(x) \mathrm{d} x .
\end{aligned}
$$


On the other hand, by (3.7), there holds

$$
\left(\rho_{+, i}^{\epsilon}(i \epsilon-, x)-\rho_{+, i}^{\epsilon}\left(i \epsilon_{+}, x\right)\right)-\left(\rho_{-, i}^{\epsilon}(i \epsilon-, x)-\rho_{-, i}^{\epsilon}\left(i \epsilon_{+}, x\right)\right)=0,
$$

from which, subtracting (3.22) from (3.21), we arrive at

$$
\int_{0}^{T} \int_{\mathbb{R}^{2}}\left(\partial_{t} \phi \rho^{\epsilon}+u^{\epsilon}\left(\rho_{+}^{\epsilon}+\rho_{-}^{\epsilon}\right) \cdot \nabla \phi\right) \mathrm{d} x \mathrm{~d} t+\int_{\mathbb{R}^{2}} \phi(0, x) \phi_{0}(x) \mathrm{d} x .
$$

(3.23) implies that $\left\{\rho^{\epsilon}\right\}$ is uniformly bounded in $\operatorname{Lip}\left(\mathbb{R}^{+}, W^{-1,1}\left(\mathbb{R}^{2}\right)\right.$ ). Note that $p>2$, by (3.18) and Lions Aubin's Lemma (see the proof Lemma 3 of [23] for a similar argument), we find that: there is subsequence $\left\{\epsilon_{j}\right\}$ and $\rho \in L^{\infty}\left(\mathbb{R}^{+}, W^{1, p}\left(\mathbb{R}^{2}\right)\right)$ such that

$$
\left\{\rho^{\epsilon_{j}}\right\} \text { uniformly converges to } \rho \text { on every compact subset } \mathbb{R}^{+} \times \mathbb{R}^{2} \text {. }
$$

With $\rho$ thus defined, we set $u=\nabla \Delta^{-1} \rho$. Then $u \in L^{\infty}\left(\mathbb{R}^{+}, W^{2, p}\left(\mathbb{R}^{2}\right)\right)$, and $\left\{u_{\epsilon_{j}}\right\}$ converges to $u$ on every compact set of $[0, \infty) \times \mathbb{R}^{2}$. Therefore from (3.23), $(\rho, u)$ is indeed a weak solution to (1.4) if we can prove that

$$
\rho_{+}^{\epsilon}+\rho_{-}^{\epsilon} \text { converges to }|\rho| \text { in } L_{\text {loc }}^{q}\left(\mathbb{R}^{+} \times \mathbb{R}^{2}\right)
$$

for any $q<\infty$. Actually by (3.2), on each time interval $[(i-1) \epsilon, i \epsilon)$, we have

$$
\partial_{t}\left(\rho_{+, i}^{\epsilon} \rho_{-, i}^{\epsilon}\right)=\left(-u_{i}^{\epsilon} \cdot \nabla \rho_{+, i}^{\epsilon}-\left(\rho_{+, i}^{\epsilon}\right)^{2}\right) \rho_{-, i}^{\epsilon}+\left(u_{i}^{\epsilon} \cdot \nabla \rho_{-, i}^{\epsilon}-\left(\rho_{-, i}^{\epsilon}\right)^{2}\right) \rho_{+, i}^{\epsilon} .
$$

Integrating the above equation over $[(i-1) \epsilon, t] \times \mathbb{R}^{2}$ with $t<i \epsilon$, and using (3.18), we obtain

$$
\int_{[-L, L]^{2}}\left(\rho_{+, i}^{\epsilon} \rho_{-, i}^{\epsilon}\right)(t, x) \mathrm{d} x \leqslant C \epsilon .
$$

Then by (3.12), (3.26) is actually for all $t \in \mathbb{R}^{+}$.

Note that

$$
\left(\rho_{+}^{\epsilon_{j}}+\rho_{-}^{\epsilon_{j}}\right)^{2}=\left(\rho^{\epsilon_{j}}\right)^{2}+4 \rho_{+}^{\epsilon_{j}} \rho_{-}^{\epsilon_{j}},
$$

which together with (3.24) and (3.26), we prove that $\left(\rho_{+}^{\epsilon_{j}}+\rho_{-}^{\epsilon_{j}}\right)^{2}$ converges to $\rho^{2}$ almost everywhere, then by Egrov Theorem, we prove (3.25).

By summing up the above argument, we achieve the following result on the global existence of strong solutions to (1.4).

Theorem 3.1. Let $\rho_{0} \in L^{1}\left(\mathbb{R}^{2}\right) \cap W^{1, p}\left(\mathbb{R}^{2}\right)$ with $2<p<\infty$. Then (1.4) has a unique global strong solution $(\rho, u)$ such that $\rho(t, x) \in L^{\infty}\left(\mathbb{R}^{+}, L^{1}\left(\mathbb{R}^{2}\right)\right) \cap L_{\mathrm{loc}}^{\infty}\left(\mathbb{R}^{+}, W^{1, p}\left(\mathbb{R}^{2}\right)\right)$, and $u(t, x) \in L_{\mathrm{loc}}^{\infty}\left(\mathbb{R}^{+}, W^{2, p}\left(\mathbb{R}^{2}\right)\right)$.

Proof. Note that the solution constructed here is strong enough, it is trivial to prove the uniqueness by comparing different solutions. We omit the details here.

When $\rho_{0} \in C^{\alpha}$ with $\alpha \in(0,1)$, or Lipschitz space, by modifying the above arguments, we can still get the global existence result to (1.4). For simplicity, we just present the result with initial data in Hölder space case.

Theorem 3.2. Let $\rho_{0} \in L^{1}\left(\mathbb{R}^{2}\right) \cap C^{\alpha}\left(\mathbb{R}^{2}\right)$ with $0<\alpha<1$. Then (1.4) has a global strong solution $(\rho, u)$ such that $\rho(t, x) \in L^{\infty}\left(\mathbb{R}^{+}, L^{1}\left(\mathbb{R}^{2}\right)\right) \cap L_{\mathrm{loc}}^{\infty}\left(\mathbb{R}^{+}, C^{\alpha}\left(\mathbb{R}^{2}\right)\right.$, and $u(t, x) \in L_{\mathrm{loc}}^{\infty}\left(\mathbb{R}^{+}, C^{1+\alpha}\left(\mathbb{R}^{2}\right)\right)$. 
Proof. With the detailed proof of Theorem 3.1, we are going to only outline the proof to the above theorem, and omit the details here.

Again as in the first step in the proof of Theorem 3.1, we can construct the approximate solutions as that in (3.12). Then we are going to get the uniform estimate of $\rho_{ \pm}^{\epsilon}$. From the proof of Theorem 3.1, we only need to get a similar version of (3.5) in the setting of Hölder space. In order to do so, let us define the plus and minus characteristics, $X_{ \pm, 1}^{\epsilon}$, by the equations

$$
\frac{\mathrm{d}}{\mathrm{d} t} X_{ \pm, 1}^{\epsilon}(t, x)= \pm u_{1}^{\epsilon}\left(t, X_{ \pm, 1}^{\epsilon}(t, x)\right)
$$

From (3.2) and (3.27), we get

$$
\frac{\mathrm{d}}{\mathrm{d} t} \rho_{+, 1}^{\epsilon}\left(t, X_{+, 1}^{\epsilon}(t, x)\right)=-\left(\rho_{+, 1}^{\epsilon}\left(t, X_{+, 1}^{\epsilon}(t, x)\right)\right)^{2},
$$

from which, we obtain

$$
\rho_{+, 1}^{\epsilon}(t, x)=\frac{\rho_{+, 0}^{\epsilon}\left(\left(X_{+, 1}^{\epsilon}\right)^{-1}(t, x)\right)}{1+t \rho_{+, 0}^{\epsilon}\left(\left(X_{+, 1}^{\epsilon}\right)^{-1}(t, x)\right)} .
$$

Let us fix a small positive constant $h$, we are going to get a uniform estimate for $\left|\left(\rho_{+, 1}^{\epsilon}(t, \cdot+h)-\rho_{+, 1}^{\epsilon}(t, \cdot)\right) / h^{\alpha}\right|$. Actually by (3.29), we get

$$
\begin{aligned}
\left|\frac{\rho_{+, 1}^{\epsilon}(t, x+h)-\rho_{+, 1}^{\epsilon}(t, x)}{h^{\alpha}}\right| & =\frac{1}{h^{\alpha}}\left|\frac{\rho_{+, 0}^{\epsilon}\left(\left(X_{+, 1}^{\epsilon}\right)^{-1}(t, x+h)\right)}{1+t \rho_{+, 0}^{\epsilon}\left(\left(X_{+, 1}^{\epsilon}\right)^{-1}(t, x+h)\right)}-\frac{\rho_{+, 0}^{\epsilon}\left(\left(X_{+, 1}^{\epsilon}\right)^{-1}(t, x)\right)}{1+t \rho_{+, 0}^{\epsilon}\left(\left(X_{+, 1}^{\epsilon}\right)^{-1}(t, x)\right)}\right| \\
& \leqslant \frac{1}{h^{\alpha}}\left|\rho_{+, 0}^{\epsilon}\left(\left(X_{+, 1}^{\epsilon}\right)^{-1}(t, x+h)\right)-\rho_{+, 0}^{\epsilon}\left(\left(X_{+, 1}^{\epsilon}\right)^{-1}(t, x)\right)\right| .
\end{aligned}
$$

While by taking $\nabla_{x}$ to (3.27), and using Gronwall inequality, we obtain

$$
\mathrm{e}^{-\int_{0}^{t}\left\|\nabla u_{1}^{\epsilon}\right\|_{L} \infty \mathrm{d} s} \leqslant\left\|\nabla_{x} X_{+, 1}^{\epsilon}\right\|_{L^{\infty}} \leqslant \mathrm{e}^{\int_{0}^{t}\left\|\nabla u_{1}^{\epsilon}\right\|_{L} \infty \mathrm{d} s} .
$$

Note that

$$
X_{+, 1}^{\epsilon}\left(t,\left(X_{+, 1}^{\epsilon}\right)^{-1}(t, x)\right)=x,
$$

by taking $\nabla_{x}$ to the above formula and using (3.31), we obtain

$$
\mathrm{e}^{-\int_{0}^{t}\left\|\nabla u_{1}^{\epsilon}\right\|_{L^{\infty}} \mathrm{d} s} \leqslant\left\|\nabla_{x}\left(X_{+, 1}^{\epsilon}\right)^{-1}\right\|_{L^{\infty}} \leqslant \mathrm{e}^{\int_{0}^{t}\left\|\nabla u_{1}^{\epsilon}\right\|_{L^{\infty}} \mathrm{d} s} .
$$

Combining (3.30) with (3.32), and taking the supremum of (3.30) with respect to $h$, we arrive at

$$
\left\|\rho_{+, 1}^{\epsilon}(t, \cdot)\right\|_{C^{\alpha}} \leqslant\left\|\rho_{+, 0}^{\epsilon}\right\|_{C^{\alpha}} \mathrm{e}_{0}^{t}\left\|\nabla u_{1}^{\epsilon}\right\|_{L^{\infty}} \mathrm{d} s .
$$

With (3.33), we can follow the proof of Theorem 3.1 step by step to complete the proof of Theorem 3.2.

\section{Acknowledgements}

Nader Masmoudi is partially supported by an NSF Grant and by an Alfred P. Sloan Fellowship. Ping Zhang is supported by NSF of China under Grant 10131050 and 10276036, the innovation grants from Chinese Academy of Sciences. The authors would like to thank Professors Fanghua Lin and Qiang Du for some discussions about the mathematical models. 


\section{References}

[1] J.S. Chapman, J.K. Rubinstein, M. Schatzman, A mean-field model of superconducting vortices, Eur. J. Appl. Math. 7 (1996) $97-111$.

[2] J.Y. Chemin, Persistance de structures géométriques dans les fluides incompressibles bidimensionnels, Ann. Sci. École Norm. Sup. 26 (1993) 517-542.

[3] R.J. DiPerna, P.L. Lions, Ordinary differential equations, transport theory and Sobolev spaces, Invent. Math. 98 (1989) $511-547$.

[4] R.J. DiPerna, P.L. Lions, On the Cauchy problem for Boltzmann equations: global existence and weak stability, Ann. of Math. (2) 130 (1989) 321-366.

[5] R.J. DiPerna, A. Majda, Oscillations and concentrations in weak solutions of the incompressible fluid equations, Comm. Math. Phys. 108 (1987) 667-689.

[6] Q. Du, P. Zhang, Existence of weak solutions to some vortex density models, SIAM J. Math. Anal. 34 (2003) 1279-1299.

[7] J.M. Delort, Existence de nappes de tourbillon en dimension deux, J. Amer. Math. Soc. 4 (1991) 553-586.

[8] C.M. Elliott, R. Schätzle, B.E.E. Stoth, Viscosity solutions of a degenerate parabolic-elliptic system arising in the mean-field theory of superconductivity, Arch. Rational Mech. Anal. 145 (1998) 99-127.

[9] L.C. Evans, Weak Convergence Methods for Nonlinear Partial Differential Equations, CBMS, vol. 74, Amer. Math. Soc., Providence, RI, 1990.

[10] R.L. Jerrard, H.M. Soner, Dynamics of Ginzburg-Landau vortices, Arch. Rational Mech. Anal. 142 (1998) 99-125.

[11] J.L. Joly, G. Métivier, J. Rauch, Focusing at a point and absorption of nonlinear oscillations, Trans. Amer. Math. Soc. 347 (1995) $3921-$ 3970.

[12] F. Lin, Some dynamical properties of Ginzburg-Landau vortices, Comm. Pure Appl. Math. 51 (1998) 323-359.

[13] F. Lin, P. Zhang, On the hydrodynamic limit of Ginzburg-Landau vortices, Discrete Contin. Dynam. Systems 6 (2000) $121-142$.

[14] P.L. Lions, Mathematical Topics in Fluid Mechanics, vol. 2, Compressible Models, Lecture Series in Mathematics and its Applications, vol. 6, Clarendon Press, Oxford, 1998.

[15] P.-L. Lions, N. Masmoudi, Global solutions for some Oldroyd models of non-Newtonian flows, Chinese Ann. Math. Ser. B 21 (2000) $131-146$.

[16] M. Schonbek, Convergence of solutions to nonlinear dispersive equations, Comm. Partial Differential Equations 7 (1982) $959-1000$.

[17] L. Tartar, Compensated compactness and applications to partial differential equations, in: Nonlinear Analysis and Mechanics: Heriot-Watt Symposium, R.J. Knops (Ed.), in: Research Notes in Math., vol. 39, Pitman, 1979.

[18] I. Vecchi, S. Wu, On $L^{1}$-vorticity for 2-D incompressible flow, Manuscripta Math. 78 (1993) 403-412.

[19] E. Weinan, Dynamics of vortex liquids in Ginsburg-Landau theories with application to superconductivity, Phys. Rev. B 50 (1994) 11261135.

[20] L.C. Young, Lectures on the Calculus of Variations and Optimal Control Theory, Saunders, Philadelphia, 1969.

[21] V.I. Yudovich, Nonstationary flow of an ideal incompressible liquid, Zh. Vych. Math. 3 (1963) 1032-1066 (in Russian). English translation: USSR Comput. Math. Phys. 3 (1963) 1407-1456.

[22] P. Zhang, Y. Zheng, Existence and uniqueness of solutions to an asymptotic equation of a variational wave equation with general data, Arch. Rational Mech. Anal. 155 (2000) 49-83.

[23] P. Zhang, Y. Zheng, Rarefactive solutions to a nonlinear variational wave equation, Comm. Partial Differential Equations 26 (2001) $381-$ 420.

[24] P. Zhang, Y. Zheng, Weak solutions to a nonlinear variational wave equation with general data, Ann. Inst. H. Poincaré Anal. Non Linéaire (2005), in press. 